\section{Case Reports in Ophthalmology}

Case Rep Ophthalmol 2017;8:459-464

DOI: $10.1159 / 000480069$

Publisned onIIne: September 14, 2017

2017 The Author(s)

Published by S. Karger AG, Basel

www.karger.com/cop

This article is licensed under the Creative Commons Attribution-NonCommercial 4.0 International License (CC BY-NC) (http://www.karger.com/Services/OpenAccessLicense). Usage and distribution for commercial purposes requires written permission.

\title{
Optical Coherence Tomography Evolution in a Case of X-Linked Juvenile Retinoschisis: 15 Years of Follow-Up
}

\author{
Irini Chatziralli ${ }^{a} \quad$ George Theodossiadis ${ }^{b}$ Dimitrios Brouzas ${ }^{c}$ \\ Ioannis Emfietzoglou ${ }^{b}$ Panagiotis Theodossiadis ${ }^{a}$ \\ a 2 nd Department of Ophthalmology, University of Athens, Attikon Hospital, \\ Athens, Greece; ${ }^{b}$ 2nd Department of Ophthalmology, Henry Dunant Hospital, \\ Athens, Greece; ${ }^{c} 1$ st Department of Ophthalmology, University of Athens, Athens, Greece
}

\section{Keywords}

Optical coherence tomography $\cdot \mathrm{X}$-linked juvenile retinoschisis $\cdot$ Children's vision

\begin{abstract}
Purpose: We present the evolution of X-linked juvenile retinoschisis (XLRS) in a male patient using optical coherence tomography (OCT) with a long-term follow-up time of 15 years. Case Description: A 10-year-old male patient presented at the Medical Retina Department of our hospital complaining for blurred vision in both eyes. At the initial presentation in 2001, his best corrected visual acuity (BCVA) was $6 / 12$ in both eyes on the Snellen chart. Based on clinical and OCT findings, the diagnosis of XLRS was made, and it was confirmed by genetic testing. No treatment was performed, but the patient was regularly examined. His BCVA and OCT findings remained relatively stable from 2001 to 2012, when BCVA decreased to 6/18 and $6 / 24$ in the right and left eye, respectively. In 2016, his BCVA was 6/24 and 6/36 in right and left eye, respectively, while OCT depicted significant macular thinning, accompanied by
\end{abstract}




\section{Case Reports in Ophthalmology}

Case Rep Ophthalmol 2017;8:459-464

(c) 2017 The Author(s). Published by S. Karger AG, Base www.karger.com/cop

Chatziralli et al.: Optical Coherence Tomography Evolution in a Case of X-Linked Juvenile Retinoschisis: 15 Years of Follow-Up

irregularities of the foveal contour in both eyes. Conclusion: Patients with XLRS should be monitored regularly to evaluate the progression of the disease and manage the potential complications.

(C) 2017 The Author(s)

Published by S. Karger AG, Basel

\section{Introduction}

X-linked juvenile retinoschisis (XLRS) is an inherited, recessive, vitreoretinal degeneration caused by mutations in the RS1 gene, encoding the retinoschisin protein $[1,2]$. Defects or the absence of this protein may lead to intraretinal microcystic development, while a coalescence of these microcysts results in retinoschisis, most commonly progressing from the outer plexiform layer or the inner nuclear layer to the inner retinal strata [2]. XLRS is more common in young males, and its prevalence is estimated to be up to 1 in 20,000 , having a bilateral appearance in $40 \%$ of the patients [1].

XLRS was first thought to arise as a result of inherited defects in müller cells. This was based on the b-wave response, which was originally thought to directly involve müller cells, and histological examinations of retina tissue from deceased patients showing filamentous material merging with müller cell membrane and splitting of the nerve fiber layer [1, 2]. However, ERG a-wave modelling has shown that phototransduction remains normal in some XLRS patients despite the reduced $b$-wave, indicating that one site of dysfunction lies beyond the photoreceptors or bipolar cells $[1,2]$.

The expression of phenotype is highly variable, but it is generally characterized by bilateral maculopathy and peripheral retinoschisis in about $50 \%$ of the patients, most often localized in the inferotemporal quadrant, which may result in retinal detachment under specific circumstances. XLRS patients usually present with decreased visual acuity when they are between 5 and 10 years old, leading to progressive visual loss during the first 2 decades of life, although stabilization of visual acuity until the fifth or sixth decade has also been observed $[1,2]$.

Clinically, patients with macular involvement in XLRS have the characteristic "spokewheel" pattern appearance of the macula on fundoscopy [1]. Peripheral retinoschisis may extend from the periphery to the macula, including the fovea, while in some cases marked retinoschisis may involve nearly the entire retina [2]. Optical coherence tomography (OCT) shows widespread cystic spaces and disorganization of the retinal layers in XLRS patients [3-5]. However, XLRS patients do not present late leakage in fluorescein angiography, since there is no suggestion of vascular involvement in XLRS [1,2].

The course of XLRS is under active investigation. Some suggest no progression, while others found that after the age of 40 the macular alterations may change from the characteristic spoke-wheel pattern to unspecific, mild, retinal pigment abnormalities [6]. In addition, during the course of the disease, secondary complications, such as retinal detachment and vitreous hemorrhage can occur, leading to poor visual outcome. However, it should be noted that vitreous hemorrhage mostly clears spontaneously and rarely needs surgery, while the results of retinal detachment surgery are usually poor even with advanced surgical techniques. Therefore, long-term monitoring might be very important. Herein, we present the evolution of XLRS in a male patient using OCT with a long-term follow-up of 15 years. 


\section{Case Reports in Ophthalmology}

\section{Case Report}

A 10-year-old-male patient presented at the Medical Retina Department of our hospital complaining of blurred vision in both eyes. At the initial presentation in 2001, the Snellen best corrected visual acuity was 6/12 in both eyes. His past medical history as well as his past ocular history were clear. There was no family history regarding eye diseases. Intraocular pressure was 14 and $15 \mathrm{~mm} \mathrm{Hg}$ in the right and left eye, respectively. Anterior segment examination was within normal limits. Fundoscopy revealed a bilateral cartwheel-like appearance of the macula in both eyes, while peripheral schisis was not apparent. OCT showed cystic lesions in the inner nuclear and outer plexiform layers of the retina, while the macular thickness was normal (Fig. 1). Based on clinical and OCT findings, the diagnosis of XLRS was made, and it was confirmed by genetic testing.

Since there was no complication of XLRS in this patient, no active treatment was recommended other than regular review. Table 1 shows the visual acuity evolution over time. There were no refractive changes over time. His visual acuity and OCT findings remained stable from 2001 to 2009 (Fig. 1, Table 1), when visual acuity decreased to 6/18 and 6/24 in the right and left eye, respectively. OCT showed coalescence and a progressive reduction in the size of the cystoid spaces, especially at the fovea. In 2016, the best corrected visual acuity was 6/24 and 6/36 in right and left eye, respectively, while OCT depicted a disappearance of the cystoid spaces and a significant macular thinning, accompanied by irregularities of the foveal contour in both eyes, along with ellipsoid zone disturbances (Fig. 1). No peripheral features of XLRS have developed during the long-term follow-up of this patient.

\section{Discussion}

The principal message of this case report is that patients with XLRS should be followed regularly, to look for complications and provide visual rehabilitation. Our case showed that patients with XLRS remain usually stable, although macular thinning was found, consistent with the decrease in visual acuity. We present a case of XLRS with a long-term follow-up time of 15 years, describing mainly the macular changes by OCT regarding size, shape, coalescence of the cystoid spaces, and ellipsoid zone integrity.

Apushkin et al. [7] postulated that a reduction in visual acuity was not associated with the size of the cystic areas or macular thickness; this is in line with Gerth et al. [8] who found that retinal layer abnormalities did not correlate with visual acuity. On the other hand, Yang et al. [9], in a retrospective study of 20 eyes with XLRS, concluded that microstructure defects of the outer plexiform layer are frequent in XLRS and may be closely related to poor vision in such patients. Other authors supported a potential mechanism for visual loss similar to agerelated macular degeneration [2]. In our case, visual acuity started to decrease when a reduction in macular thickness was observed, followed by the coalescence of the cystoid spaces. The progression to macular atrophy and visual impairment was associated with ellipsoid zone disturbance. It is also worthy to note that the evolution of the disease was similar in both eyes. Regarding the mechanism for retinal thinning, we hypothesize that the collapse of the cystoid spaces may result in a remodeling and loss of cellular tissue, which lead to retinal thinning, but further investigation is needed to examine the exact pathogenesis. 
In conclusion, we describe the case of a male patient with XLRS, showing the evolution of XLRS using OCT scans. The disease was characterized by the presence of cystoid spaces with almost similar appearance in both eyes. We observed a progressive coalescence of the cystoid spaces which led to macular atrophy with consequent visual impairment during a 15-year follow-up. The visual acuity also progressively deteriorated from 6/12 to 6/24 during the follow-up period. Therefore, patients with XLRS should be monitored regularly to evaluate the progression of the disease and manage the potential complications.

\section{Statement of Ethics}

Written informed consent was obtained from the patient for reporting the case.

\section{Disclosure Statement}

All authors declare no conflict of interest and no financial disclosure.

\section{References}

1 Kim DY, Mukai S: X-linked juvenile retinoschisis (XLRS): a review of genotype-phenotype relationships. Semin Ophthalmol 2013;28:392-396.

2 Molday RS, Kellner U, Weber BH: X-linked juvenile retinoschisis: clinical diagnosis, genetic analysis, and molecular mechanisms. Prog Retin Eye Res 2012;31:195-212.

-3 Saxena S, Manisha, Meyer CH: Three-dimensional spectral domain optical coherence tomography in X linked foveal retinoschisis. BMJ Case Rep 2013;2013.

-4 Ozdemir H, Karacorlu S, Karacorlu M: Optical coherence tomography findings in familial foveal retinoschisis. Am J Ophthalmol 2004;137:179-181.

5 Brucker AJ, Spaide RF, Gross N, Klancnik J, Noble K: Optical coherence tomography of X-linked retinoschisis. Retina 2004;24:151-152.

6 Urrets-Zavalía JA, Venturino JP, Mercado J, Urrets-Zavalía EA: Macular and extramacular optical coherence tomography findings in X-linked retinoschisis. Ophthalmic Surg Lasers Imaging 2007;38:417-422.

7 Apushkin MA, Fishman GA, Janowicz MJ: Correlation of optical coherence tomography findings with visual acuity and macular lesions in patients with $\mathrm{X}$-linked retinoschisis. Ophthalmology 2005;112:495-501.

8 Gerth C, Zawadzki RJ, Werner JS, Héon E: Retinal morphological changes of patients with X-linked retinoschisis evaluated by Fourier-domain optical coherence tomography. Arch Ophthalmol 2008;126:807-811.

-9 Yang HS, Lee JB, Yoon YH, Lee JY: Correlation between spectral-domain OCT findings and visual acuity in X-linked retinoschisis. Invest Ophthalmol Vis Sci 2014;55:3029-3036. 


\section{Case Reports in Ophthalmology}

Case Rep Ophthalmol 2017:8:459-464

DOI: $10.1159 / 000480069$

(C) 2017 The Author(s). Published by S. Karger AG, Basel www.karger.com/cop

Chatziralli et al:: Optical Coherence Tomography Evolution in a Case of X-Linked Juvenile Retinoschisis: 15 Years of Follow-Up
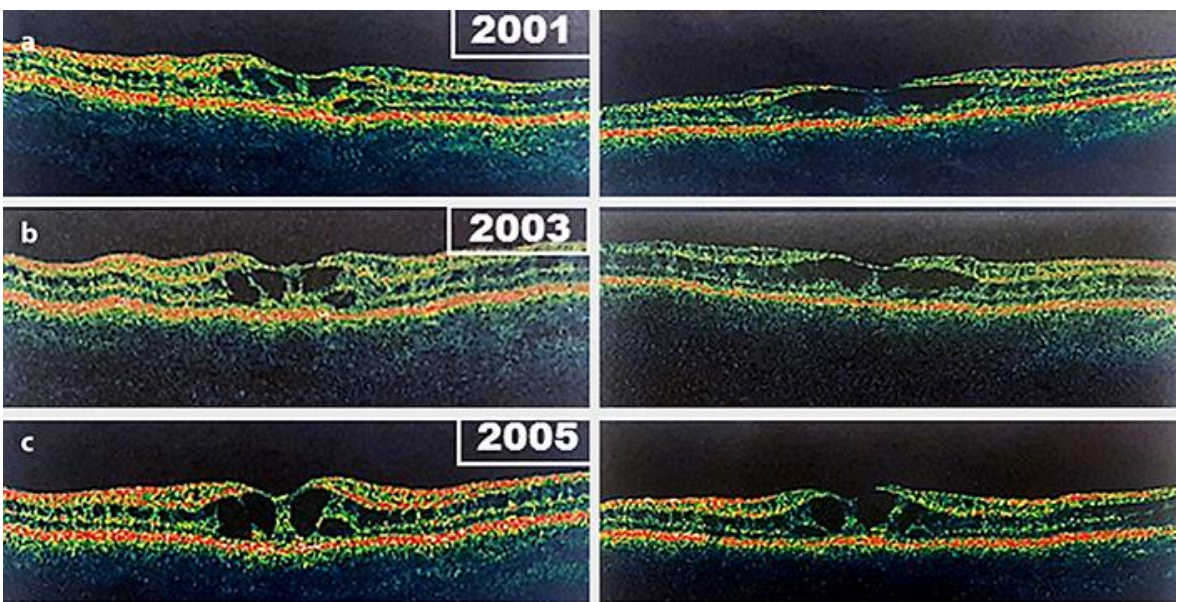

d

2007
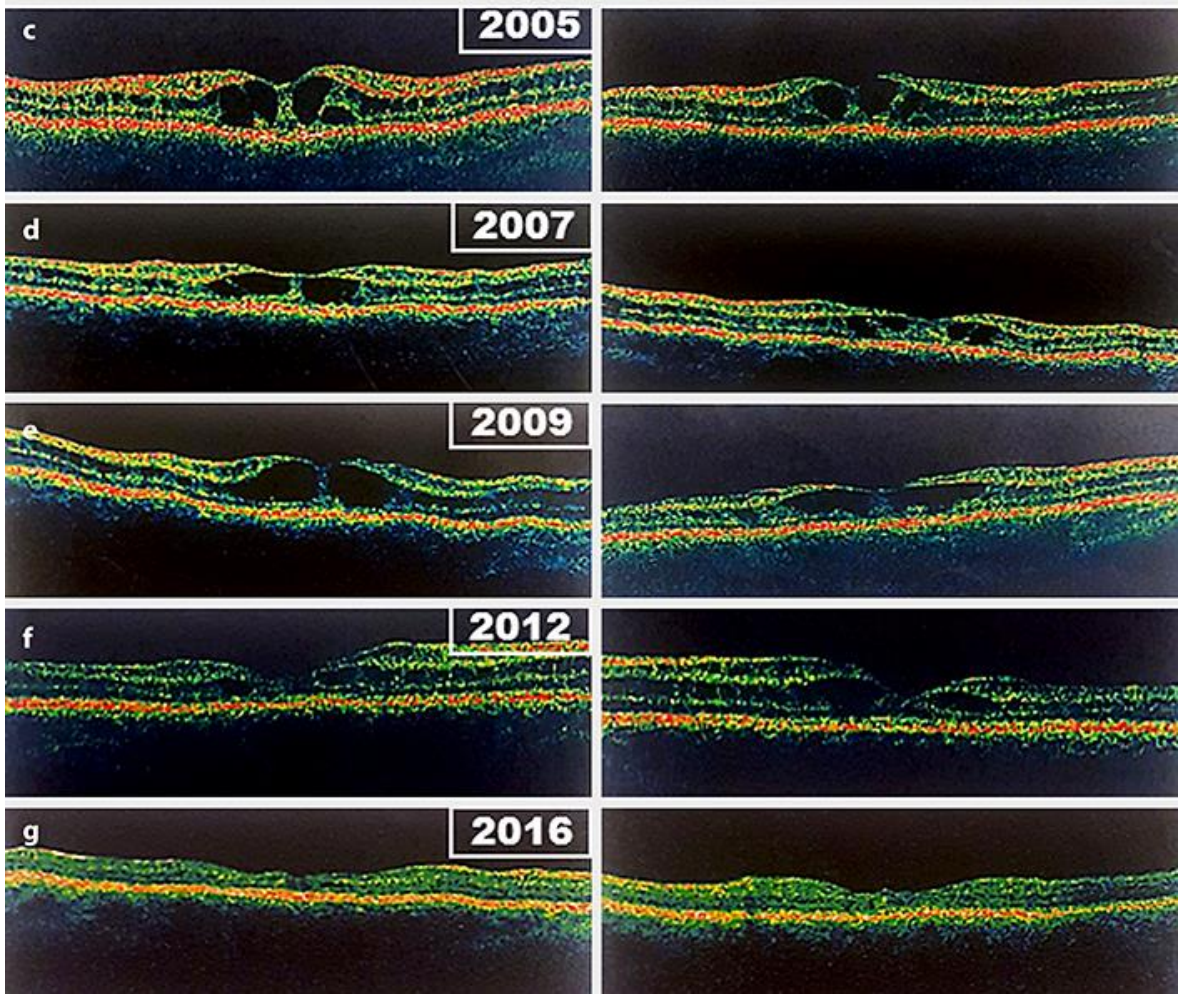

Fig. 1. Evolution of the optical coherence tomography findings with time in the right (left panel) and left eye (right panel), respectively, in a male patient with X-linked juvenile retinoschisis. a, b Presence of cystoid spaces at the macular area. c-e Coalescence of the cystoid spaces and progressive reduction in macular thickness. $f$ Retinal thinning, reduction of the cystoid spaces in number and size, and disturbance of ellipsoid zone integrity. $g$ Disappearance of the cystoid spaces, significant decrease in macular thickness, irregularity of the foveal contour, and attenuation of the ellipsoid zone. 
Chatziralli et al.: Optical Coherence Tomography Evolution in a Case of X-Linked Juvenile Retinoschisis: 15 Years of Follow-Up

Table 1. Evolution of our patient's best corrected visual acuity over time

\begin{tabular}{lll}
\hline \multirow{2}{*}{ Year } & \multicolumn{2}{l}{ Best corrected visual acuity (Snellen) } \\
\cline { 2 - 3 } & Right eye & Left eye \\
\hline 2001 & $6 / 12$ & $6 / 12$ \\
2003 & $6 / 12$ & $6 / 12$ \\
2005 & $6 / 12$ & $6 / 9$ \\
2007 & $6 / 12$ & $6 / 12$ \\
2009 & $6 / 12$ & $6 / 12$ \\
2012 & $6 / 18$ & $6 / 24$ \\
2016 & $6 / 24$ & $6 / 36$ \\
\hline
\end{tabular}

